\title{
IL DIRITTO SOCIETARIO TRA DIRITTO COMPARATO E DIRITTO STRANIERO
}

\author{
Nota del m.e. GIUSEPPE B. PORTALE (*)
}

(Adunanza del 7 marzo 2013)

SunTO. - È stato scritto da un autorevole storico del diritto (GROSSI, Il diritto) che «Prima, il diritto era costretto all'ombra dello Stato e tendeva ad identificarsi in un complesso di leggi; ora, va riscoprendo la sua fisiologica dimensione di ordinamento dell'intera società e chiede di manifestarsi a delle fonti plurali, che, accanto alla legge, sono le consuetudini, le sentenze dei giudici, le opinioni autorevoli della scienza giuridica. Se il diritto moderno - settecentesco e ottocentesco - era all'insegna della semplicità (una semplicità indubbiamente non spontanea, ma forzosa, e quindi artificiosa), quello novecentesco ed odierno, misurandosi ormai liberamente sulla società, si presenta in tutta la sua complessità. - Prima, un rigido monismo giuridico; nell'immediato ieri e nell'oggi, un aperto pluralismo giuridico. Prima, solo il grande burattinaio dello Stato nazionale; nell'immediato ieri e nell'oggi, anche l'Europa e il fattualissimo ma effettivo diritto globale tutto modellato sulla fattualità economica emergente. All'interno dello Stato nazionale, non solo leggi, ma una pluralità di fonti che ne valga a colmare l'incapacità del legislatore a disciplinare il magma che stiamo vivendo. - Nuovi protagonisti fanno, ormai, opera di supplenza, e soprattutto i giudici, personaggi di trincea, dai quali il cittadino comune reclama giustizia per il caso concreto della sua vicenda quotidiana. E si profila anche - e sempre più decisamente - un nuovo assetto delle fonti ormai plurali ... ». Il tema che le superiori riflessioni mi hanno suggerito, per questo breve intervento, è quello del diritto comparato come fonte del diritto in un particolare settore del nostro sistema: specificamente, quello del diritto nazionale (o domestico) delle società di capitali. A detto tema - soprattutto, negli ultimi decenni - se ne è affiancato un secondo, al quale, fino a tempi recenti, era stata dedicata - quanto meno nel nostro ambiente giuridico - assai scarsa attenzione: quello dell'applicazione del diritto societario straniero da parte del giudice italiano (v. ora, LA MATTINA; nella letteratura

(*) Prof. em. (Diritto commerciale) dell'Università Cattolica di Milano, Italy. E-mail: studio@portalevisconti.it 
tedesca: THOLE). A questi argomenti - che presuppongono precise posizioni di vertice, non limitate al diritto societario, né correlate alla sola problematica dell'autonomia del diritto commerciale (da ultimi, LiBERTINI, e DELLE MONACHE) - farò seguire, a scopo applicativo, dei cenni a qualche fattispecie di nuovo conio.

$* * *$

ABSTRACT. - The article tackles the issues related to the use of comparative law a source of substantive law in a specific legal system, with specific regard to corporate law. Expanding on previous studies on the general role of comparative law in the framework of sources of law $(\$ 1)$, the study argues that the comparative argument may be used to regulate purely domestic cases and as well as a play a crucial role in interpreting internal laws $(\mathbb{S} 1.1,1.2)$ and analyzes the theoretical foundations of such process $(\mathbb{1 . 3}$ ) as well as the problems caused by the application of foreign law by a domestic judge ( $\$ 2)$. Subsequently, two examples of such usage of the comparative legal argument are provided, drawn from the Italian corporate law experience $(\$ 3)$ : on the one hand, the introduction of a specific regulation of a simplified private company (società a responsabilità limitata semplificata), representing a circulation of German (Unternebmergesellschaft$U G$ ) and Belgian (société privée a responsabilité limitée starter) models $(\$ 3.1)$; on the other hand, the use of comparative law by in the interpretation of the organization structure in the Italian dualistic system $(\mathbb{3} .2)$.

1. La moderna dottrina comparatistica alle varie funzioni attribuite al diritto comparato - in particolare, oltre a quelle di trovare e preparare materiali che possano successivamente servire ai legislatori nazionali (e, al proposito, per quanto riguarda il nostro paese è sufficiente ricordare i nuovi istituti recepiti dalla riforma delle società di capitali del 2003 e dal c.d. t.u.f. del 1998 e dalle successive modifiche) oppure per la redazione delle direttive comunitarie o di leggi uniformi (ad esempio, sempre per limitarmi al diritto societario, i Regolamenti CE dell'8 ottobre 2001, n. 2157, e del 22 luglio 2003, n. 1435, dedicati, rispettivamente, alla società europea [SE] e alla società cooperativa europea [SCE]) -, aggiunge il compito di strumento per l'interpretazione e la ricostruzione del diritto interno, specialmente, in relazione agli istituti trapiantati da altri sistemi, ma anche per colmare lacune: e tutto questo pur in mancanza di una norma equivalente a quella dell'art. 1, comma 2, cod. civ. svizzero («nei casi non previsti dalla legge, il giudice decide secondo la consuetudine e, in difetto di questa, secondo la regola che egli adotterebbe come legislatore»).

È subito da dire che la questione non è nuova (di essa si discute fin dalla fine del secolo XIX: GOLDSCHMIDT e KOHLER; per un lucido schizzo storico v., con posizione cauta sull'attuale funzione della comparazione 
giuridica, RANIERI) e che non attiene solamente al c.d. diritto europeo, ovvero all'esistenza di uno ius commune europaeum. Rileva molto più in generale il ricorso alle disposizioni di un ordinamento altro rispetto all'ordinamento italiano o di altro paese dell'Unione europea, come può accadere in collegamento con la ricezione di istituti sbocciati nel diritto statunitense (cfr. la monografia di von HeIN; per un istruttivo catalogo di istituti e figure anglo-americani che hanno trovato accoglienza nel diritto azionario tedesco, FLEISCHER), anche se, evidentemente, all'interno del problema civilcommercialistico dell'interpretazione, uno spazio privilegiato, nel ricorso alla lex alii loci, deve essere riservato al diritto europeo, come dimora attuale del nostro diritto (SOMMA, molto incisivi e documentati, anche, Monateri/Somma; adde: Pizzorusso e von Hein). Va ulteriormente precisato e sottolineato - come già implicitamente dovrebbe risultare - che i richiami al diritto straniero di cui qui discuto ricorrono pure in fattispecie interamente disciplinate dal diritto interno, in cui il richiamo in questione diviene in un certo senso facoltativo o comunque cessa di essere «internazionalmente» obbligato (SOMMA; ALPA): come accade allorché la fattispecie sottoposta al giudice o di cui si occupa l'interprete non presenti elementi di estraneità rispetto al diritto italiano (perché, diversamente, si ricadrebbe nell'ambito di applicazione dell'art. 14 l. n. 218/1995). Si allude, qui, alla ipotesi in cui l'esito della decisione del giudice nostrano o acquisito dall'interprete utilizza il diritto straniero (talvolta, attraverso il filtro della relativa giurisprudenza: SOMMA; GAMBARO; ALPA) - non solo come strumento idoneo ad assecondare processi di ravvicinamento delle legislazioni o di unificazione internazionale delle norme (in questa direzione già ZiTELMANN) ma anche - per promuovere e avvalorare le finalità di volta in volta avute in mente dallo stesso interprete (non a caso, si parla, allora di «uso normativo della comparazione»: SMORTO). Viene soprattutto in considerazione - è stato scritto - il proposito di provocare l'evoluzione giurisprudenziale del diritto (Rechtsfortbildung), dove per giurisprudenza deve intendersi sia quella teorica sia quella empirica (ampiamente, SOMMA).

Due esempi possono ben chiarire il discorso:

a) con il primo, va fatta rimarcare la presa di posizione, sia di parte rilevante della nostra dottrina che della giurisprudenza, a favore dell'applicabilità (prima e a prescindere dall'introduzione dell'art. 182quater nella legge fallimentare) alle società per azioni (quanto meno a quelle chiuse) dell'istituto della c.d. postergazione dei finanziamenti dei soci a società materialmente sottocapitalizzate, disciplina- 
to con riferimento alla società a responsabilità limitata. È significativo che nelle motivazioni delle sentenze delle nostre Corti si utilizzino (e questo mi sembra l'epifania dell'esistenza di una concorrenza degli ordinamento anche all'interno dell'Unione Europea dove - in materi societaria - non è stato raggiunto e, forse, non lo sarà mai un ravvicinamento delle legislazioni a livello globale: ZOPPINI, PORTALE, Le nuove norme) espressioni che si leggono nelle motivazioni delle decisioni e nei contributi degli scrittori germanici: come quella di «sottocapitalizzazione nominale» o quella che l'art. 2467 c.c. «esprime un principio generale di corretto finanziamento dell'impresa»; e lo stesso accade quando le decisioni dei giudici italiani riportano al «concetto normativo di "finanziamenti" in qualsiasi forma effettuati» le «fideiussioni e garanzie del socio a favore della società, prestate in un particolare momento di indebitamento della società» (v., tra i tanti, Trib. Padova, 16 maggio 2011; Trib. Venezia, 8 marzo 2011; Trib. Udine, 3 marzo 2009, con commento di BALP).

b) Pur senza trascurare l'ammonimento di FRANZ WIEACKER - ben presente a FLEISCHER - che gli istituti stranieri trapiantati hanno sempre una propria evoluzione in simbiosi con il sistema del paese di recezione, di tal ché diventano elementi della sua vita e della sua cultura, il secondo esempio può essere concretizzato con la faticosa ricostruzione - che implica la necessità di colmare alcune vistose lacune della normativa italiana - della disciplina del c.d. modello dualistico di amministrazione e controllo (importato dal diritto azionario tedesco), con riferimento al quale mi limito a richiamare il recente volume di CARIELlo (a cui si deve anche un meditato scritto sull'uso della comparazione in materia di gruppi di società).

1.2. La risposta positiva al problema della trasportabilità della soluzione straniera come strumento di sviluppo del diritto italiano trova, allo stato, due giustificazioni (fondamenti) alternative. La prima di esse è suggerita dai cultori di diritto comparato (cfr. già ZWEIGERT; da noi MONATERI/SOMMA, con rinvio a GORLA), i quali reputano che «il richiamo al diritto comparato [debba] ... avvenire nell'ambito delle "opzioni interpretative", cioè nell'ambito del $2^{\circ}$ comma dell'art. 12 Preleggi» (v., anche, Rescigno; Somma, Metodi e scopi; critico, SMORTO). Specificamente, attraverso l'esegesi di detto comma («se una controversia non può essere decisa con una precisa disposizione, si ha riguardo alle disposizioni che regolano casi simili o materie analoghe; se 
il caso rimane ancora dubbio, si decide secondo i principi generali dell'ordinamento giuridico dello Stato»), i comparatisti sostengono che il ricorso all'interpretazione analogica - a differenza di quanto accade per il criterio residuale del ricorso ai principi generali dell'ordinamento giuridico - non è vincolato alle sole norme che compongono l'ordinamento giuridico dello Stato. E tutto questo è accaduto - si aggiunge: sulla base dei lavori preparatori del medesimo secondo comma dell'art. 12 delle Preleggi - proprio per consentire l'applicazione analogica anche di materiali normativi stranieri.

La seconda giustificazione della legittimità del ricorso al diritto comparato - come strumento per colmare le lacune del sistema - appare più moderna e più convincente: essa si basa su un discorso di vertice, che risulta comune ai teorici generali del diritto, ai cultori del diritto pubblico, agli internazionalisti, agli storici del diritto e ai giuscommercialisti, come è ben emerso in un Convegno sulle fonti del diritto presso l'Accademia Nazionale dei Lincei (19 aprile 2012), in occasione della presentazione della nuova edizione dell'opera di Pizzorusso sull'argomento. Punto di partenza degli studiosi dei predetti settori è che (appare già dalle parole di Paolo Grossi riportate in apertura) il sistema delle fonti elencate nell'art. 1 delle Preleggi - dopo la frammentazione avvenuta sia a livello di codice civile che a livello dei diritti settoriali, e i ridimensionamenti patiti dai nostri diritti nazionali, tanto da apparire modificato il c.d. sistema di Vestfalia (1646) -, è da reputare del tutto superato, per la necessità di dover ammettere l'esistenza di altre fonti e di dover riconoscere l'incompletezza di qualsiasi codice che dichiarasse tassativo il proprio sistema delle fonti. Senza potermi attardare sul punto, questo orientamento sostiene che «all'area del diritto giurisprudenziale ... vanno ricondotti [anche] i fenomeni che portano all'impiego di regole giuridiche comuni nell'ambito di ordinamenti diversi, non tanto per effetto di espliciti atti di ricezione di testi normativi già vigenti altrove come tali, quanto per lo spontaneo riconoscimento dell'idoneità di un principio o di una regola a valere come norma giuridica anche fuori dell'area con riferimento alla quale essa è stata dapprima adottata, o comunque in modo indipendente da una sua formale adozione come tale» (Pizzorusso, 739).

Segnatamente, si allude in questo modo al c.d. «diritto transnazionale», inteso qui in un significato diverso dalla c.d. nuova lex mercatoria (sulla quale, fra molti, GALGANO; per un giudizio disincantato, GROSSI, Globalizzazione), in quanto si chiarisce che in questo contesto il sintag- 
ma «diritto transnazionale [esprime] l'insieme di quei principi giuridici fondamentali che appaiono suscettibili di essere accettati nei diversi ordinamenti, anche indipendentemente dal fatto di essere in essi introdotti attraverso espliciti atti normativi, perché corrispondenti ad una concezione del diritto fondata sulla ragione e comune a tutti gli uomini e a tutti i popoli, o quantomeno ai popoli appartenenti ad una certa area culturale» (Pizzorusso, 742).

1.3. Ed è proprio quest'ultima proposizione che aiuta a risolvere - per entrambi gli orientamenti esposti - l'ulteriore questione (definita preliminare) dell'accertamento della disposizione straniera applicabile (in via diretta o in via analogica) alla controversia. Al proposito, è stato scritto che - non essendo nel nostro caso, come più volte precisato, l'impiego del diritto straniero prescritto da una norma di diritto internazionale privato -, «la scelta dell'ordinamento da cui ricavare la disposizione straniera si lega a due esigenze. Da un lato occorre individuare esperienze giuridiche in cui siano riconosciuti valori che l'interprete intende promuovere. Dall'altro bisogna dimostrare che esiste una vicinanza possibilmente notevole fra quelle esperienze e quella dell'ordinamento candidato a ricevere il modello straniero» (MONATERI/SOMMA). In questo senso, si diceva, fino a qualche decennio $\mathrm{fa}$, che sarebbe dovuto valere, innanzitutto, il ricorso al diritto francese e a quello tedesco ed ai sistemi da loro derivati, secondo gradi di lontananza della nostra esperienza; ma oggi non può negarsi che è entrato prepotentemente in scena anche il diritto anglo-americano (SomMA, L'uso giurisprudenziale, 287 ss.; Grossi, Globalizzazione; von HeIN).

2. Passando al secondo argomento oggetto di queste note - e cioè l'applicazione da parte di un giudice nazionale del diritto societario straniero -, devo dire che esso sta trovando un discreto interesse: specialmente, nella dottrina e nella giurisprudenza tedesche. E tutto questo in conseguenza delle note sentenze della Corte di Giustizia (ricordo, per tutte, le sentenze Centros, Überseering e, soprattutto, Inspire Art) sulla libertà di stabilimento, che hanno anche prodotto l'affermarsi della c.d. Gründungstheorie (teoria della costituzione), il cui effetto è stato quello della migrazione dell'attività di oltre 50.000 Limited private Companies inglesi in territorio tedesco, restando soggette al diritto del luogo di costituzione, e cioè al diritto inglese, assai lasco in materia di formazione del capitale sociale e di conferimenti in natura, 
in quanto questo tipo di società non è sottoposta alla II Direttiva comunitaria in materia societaria (77/91/CEE): dato che essa concerne solo le società per azioni. Il problema può dirsi che si pone allo stesso modo sia nel nostro diritto che in quello tedesco: il $\$ 293 \mathrm{ZPO}$, nella sostanza, corrisponde, infatti, all'art. 14 della nostra 1.31 maggio 1995 , n. 218, a norma del quale l'accertamento della legge straniera è compiuto d'ufficio dal giudice (primo comma); la stessa disposizione stabilisce, altresì, che «qualora il giudice non riesca ad accertare la legge straniera indicata, neanche con l'aiuto delle parti, applica la legge richiamata mediante altri criteri di collegamento eventualmente previsti per la medesima normativa. In mancanza si applica la legge italiana» (secondo comma; per l'esame: BARATTA; CARBONE; IVALDI). Entrambe le diposizioni quella italiana e quella tedesca - confermano, così, la regola «iura novit curia», che, in questo caso, più correttamente, diventa «iura aliena novit curia», con il risultato che il diritto straniero non rileva più - così accadeva in passato - come fatto, sfuggendo in tal modo al sindacato del giudice di legittimità, bensì proprio come diritto, suscettibile, pertanto, del controllo della Corte Suprema (art. 360, comma 1, n.3, c.p.c. it.; $\$ 545$ ZPO: BARATTA; IVALDI; Cass., sez. lav., 5 giugno 2009, n. 13087; Cass., 9 maggio 2007, n. 1059; Cass., 26 febbraio 2002, n. 2791).

Gli studiosi tedeschi precisano, condivisibilmente, che, nell'oggetto dell'accertamento di "queste norme giuridiche", rientra anche l'interpretazione e l'applicazione che ne fanno i giudici stranieri attraverso la giurisprudenza straniera (THOLE). Il Bundesgerichtshof (BGH, 27 aprile 1976) si esprime in questi termini: «a norma del $\$ 293 Z P O$, il giudice è obbligato ad accertare il diritto straniero sfruttando tutte le fonti di conoscenza a lui accessibili; egli non deve basarsi solo sulle norme positive, ma deve anche tenere conto di come queste, sul piano dell'effettività, vengono conformate dalla dottrina e dalla giurisprudenza». E a queste precisazioni, fa seguito l'ammonimento che la sentenza tedesca deve risultare vicina a quella che sarebbe stata emessa nel paese della lex causae, restando, pertanto, chiaro che il diritto straniero non viene recepito, ma è solo da applicare.

Senza potere soffermarmi né sulle implicazioni dell'applicazione da parte di un giudice di civil law (e, quindi, anche da parte di un giudice italiano) della common law, né sull'obbligo dello stesso giudice di disapplicare l'eventuale norma di un paese dell'Unione europea che sia in contrasto con una Direttiva comunitaria, per applicare direttamente la norma europea, desidero concludere il tema con due assunti della 
dottrina germanica in materia societaria. Secondo il primo, l'applicazione degli strumenti interni di tutela dei creditori è coperta nei confronti della libertà di stabilimento soltanto nel caso in cui il diritto del luogo di costituzione della società non offre alcuna equivalente tutela ai creditori. In base al secondo assunto «lo statuto della società (straniera), nella sostanza, comprende la costituzione, la capacità giuridica e lo scioglimento delle società, come pure la responsabilità personale dei soci e degli organi sociali per i debiti della società», mentre resterebbero esclusi - ma il punto, a mio giudizio, dovrebbe essere meglio approfondito - altri istituti: quali la c.d. Existenzvernichtungshaftung (responsabilità dei soci per abusi, a danno dei creditori sociali, sul patrimonio della società, provocandone o aggravandone l'insolvenza: HÜFFER, $\$ 1, R d n$. 22), la revocatoria dei finanziamenti soci, come pure la responsabilità dei gestori per la ritardata richiesta del fallimento ex $\$ 64$ GmbHG (THOLE).

3. Mi ero riservato, in funzione applicativa, di dedicare dei cenni a qualche fattispecie di nuova introduzione nel nostro diritto delle società di capitali.

A) Desidero, anzitutto, richiamare le due subfattispecie di società a responsabilità limitata, che sono il prodotto dei due c.d. «decreti sviluppo» del 2012 (d.1. 24 gennaio 2012, n. 1, convertito nella 1. 24 marzo 2012, n. 27, e d.1. 22 giugno 2012, n. 83, convertito nella 1. 7 agosto 2012, n. 134), i quali, rispettivamente, hanno introdotto la «società a responsabilità semplificata» (art. 2463-bis c.c.), caratterizzata, sul piano soggettivo, dall'essere la sua costituzione riservata «a persone fisiche che non abbiano compiuto i trentacinque anni di età» e la «società a responsabilità limitata a capitale ridotto» (art. 44 d.l. n. 83/2012), caratterizzata anch'essa dall'essere riservata a persone fisiche, ma senza alcun limite di età. Il dato oggettivo principale «tipizzante» di entrambe le subfattispecie va individuato nella disposizione che l'ammontare del capitale sociale deve essere «pari almeno ad 1 euro e inferiore all'importo di 10.000 euro», che corrisponde, come è noto, al capitale minimo stabilito per la società a responsabilità limitata (per così dire) «normale» (art. 2463, comma 1, n. 4, c.c.). Tralasciando di criticare la rozzezza della tecnica legislativa (si parla, tra l'altro, di versamento del conferimento all' «organo amministrativo», che secondo l'opinione corrente non può esistere prima dell'iscrizione della società nel registro delle imprese), e di indagare su qualche dubbio di costituzionalità cui si espongono i due «subtipi» (non si comprende, ad esempio, 
perché, a questo punto, l'imprenditore individuale debba restare esposto alla responsabilità illimitata: art. 2740 c.c.), come pure se entrambe le subfattispecie siano effettivamente idonee - soprattutto, in tempo di crisi ed in prossimità dell'entrata in vigore di Basilea 3 -, ad incentivare la creazione di nuove attività imprenditoriali da parte di giovani ed in genere da parte di persone fisiche senza patrimonio, è qui da sottolineare l'assenza di una congrua disciplina su punti fondamentali implicati dai due «subtipi» (sui quali, anche per la documentazione: CIAN; DACCOे). Solo per indicare qualche criticità: incerto è, in primo luogo, se e fino a che punto sia derogabile il «modello standard» per la loro costituzione, «tipizzato con decreto del Ministro della giustizia, di concerto con il Ministro dell'economia e delle finanze e con il Ministro dello sviluppo economico» (art. 2463-bis, comma 2, c.c. richiamato dall'art. 44 del d.l. n. 83/2012); ugualmente, incerta è la portata del divieto di effettuare conferimenti in natura (art. 2463-bis, comma 2, n. 3: a contrario), come anche resta incerto se - qualora il c.d. patrimonio netto raggiunga o superi l'importo di 10.000 euro - sussista un obbligo di adeguare lo statuto a quello di una società a responsabilità limitata «normale», e se questo transito alla società «normale» debba essere qualificato come trasformazione (art. 2498 ss. c.c.), con tutte le relative conseguenze. E rimane pure da chiarire se possa (o debba) trovare applicazione l'art. 2467 c.c. sul finanziamento dei soci (v. supra, n. 1, sub a), ed ancora se sia configurabile, quanto meno in certi casi, una responsabilità dei soci da Durchgriff (=superamento della personalità giuridica della società) o per fatto illecito nei confronti dei creditori sociali (specificamente: quelli «deboli» o quelli c.d. «involontari»). Dal momento che con l'introduzione delle due subfattispecie il nostro legislatore ha voluto imitare, mi consta personalmente, esperienze straniere - in particolare: la c.d. «Unternehmergesellschaft» (=società per piccoli imprenditori) tedesca introdotta con il $\$ 55$ a $G m b H G$ e la sociétéprivée à responsabilitè limitée starter belga disciplinata dagli artt. 211-bis e 241 code soc. (von DeR LAAGE; PORTALE; CIAN) -, reputo che la rimarcata insufficienza della nostra disciplina possa (e, forse, debba) essere supplita, nelle sue lacune, attingendo (non sono in grado di valutare se con una qualche abbondanza) alla elaborazione dottrinale e giurisprudenziale di cui già fruiscono i due richiamati modelli stranieri.

B) Sempre in funzione applicativa, concludo accennando, con rapidità, ad una questione che, da ultimo, si è posta nel disegnare i limiti di competenza dell'assemblea nelle società per azioni con sistema 
dualistico di amministrazione e controllo (dietro, n. 1, lett. $b$ ): anch'essa risolta, quanto meno da una parte degli scrittori domestici, integrando la nostra disciplina con normative straniere (ABBADESSA/MIRONE; PORTAle, Amministrazione). Il quesito è se all'assemblea possa essere riconosciuta una funzione di «arbitraggio» nei conflitti gestionali tra consiglio di gestione e consiglio di sorveglianza. Un siffatto ruolo è in effetti attribuito all'assemblea dal $\$ 111, A b s .4, S .3, A k t G$ tedesco, con riguardo a tutte le società per azioni. La norma - malgrado in Germania, probabilmente, non abbia mai trovato applicazione: per evitare gli effetti negativi (soprattutto, con riguardo alle società quotate) del rendere pubblico l'essere venuta meno la fiducia tra consiglio di sorveglianza e consiglio di gestione -, è stata riprodotta in molti altri sistemi europei (da ultimo, in Lussemburgo, dove l'art. 60-bis-7, $\$ 2$, comma 2 , della legge sulle società commerciali, stabilisce che «lorsqu'une opération exige l'autorisation du conseil de surveillance et que celui-ci la refuse, le directoire peut soumettre le différend à l'assemblée générale»). Per quanto - in occasione della riforma del diritto delle società di capitali del 2003 -, dei due suggerimenti sul punto da me trasmessi alla c.d. «Commissione Vietti» ne sia stato accolto solo uno (art. 2409-terdecies, comma 2, c.c.: mancata approvazione del bilancio), per di più mitigato (necessaria previsione statutaria: v., per contro, $\mathbb{} 173 \mathrm{Akt} G$ tedesco), omettendo l'introduzione di una norma generale come quella codificata nel citato $\$ 111, A b s$. 4, Akt $G$ ed ora anche nel diritto lussemburghese, non credo che detta omissione possa impedire di colmare la lacuna. E questo non solo perché nemmeno dai lavori preparatori della riforma societaria del 2003 si traggono controindicazioni, quanto perché, appunto, bisogna ormai ammettere - specialmente in certi settori del diritto - la trasportabilità delle soluzioni straniere come strumento di sviluppo dello stesso.

\section{RIFERIMENTI BIBLIOGRAFICI}

P. AbBadessa e A. Mirone, Le competenze dell'assemblea nelle s.p.a., in Riv. soc., 2010, 300.

G. ALPA, Il giudice e l'uso delle sentenze straniere. Modalità e tecniche della comparazione giuridica. La giurisprudenza civile, nel volume collettaneo con il medesimo titolo, curato dallo stesso G. Alpa, Milano, 2006, 33.

G. BALP, Questioni in tema di postergazione ex art. 2467 e 2497 -quinquies c.c., in Banca, borsa, tit. cred., 2012, II, 246. 
R. BARATTA, Diritto straniero (accertamento e conoscenza), in Dizionari del diritto privato, promossi da N. IRTI. Diritto internazionale privato, a cura di R. Baratta, Milano, 2010, 147.

S.M. CARBOnE, La conoscenza del diritto straniero e il giudice italiano, in Dir. comm. int., 2009, 193.

V. CARIEllo, Il sistema dualistico, Torino, 2012; ID., Sensibilità comuni, uso della comparazione e convergenze interpretative: per una Methodenlehre unitaria nella riflessione europea sul diritto dei gruppi di società, in Riv. dir. soc., 2012, 255.

M. CiAN, S.r.l. semplificata, s.r.l. a capitale ridotto. Una nuova geomertria del sistema o un sistema disarticolato?, in Riv. soc., 2012, 1101.

A. DACCÒ, La società a responsabilità limitata semplificata e la società a responsabilità limitata a capitale ridotto, in Diritto commerciale aggiornato alla legge 11 agosto 2014, n. 116, a cura di M. Cian, Torino, II, 2014, sez. IV.

S. Delle Monache, "Commercializzazione" del diritto civile (e viceversa), in Riv. dir. civ., 2012, I, 495.

G.D. von DER LAAGE, La "Unternehmergesellschaft (haftungsbeschränkt)": il nuovo modello di $\mathrm{GmbH}$ (s.r.l.) nella recente riforma tedesca, in Riv. soc., 2011, 404.

H. FLEISCHER, Legal Transplants im deutschen Aktienrecht, in NZG, 2004, 1129.

F. GALGANO, Lex mercatoria, Bologna, 2010.

A. Gambaro, Il diritto comparato nelle aule di giustizia ed immediati dintorni, in AA.Vv., L'uso giurisprudenziale della comparazione giuridica, Milano, 2004, 3.

L. GOLDSCHMIDT, Buchbesprechung, in ZHR, 1882, 451.

P. GROSsI, Il diritto nella storia dell'Italia unita: reperibile in www.lincei .it/files/convegni/840-allegatouno.pdf; ID., Globalizzazione, diritto, scienza giuridica, in Atti Accademia Nazionale dei Lincei, CCCIC - 2002 - Classe di Scienze Morali, Storiche e Filologiche. Rendiconti - Serie IX - vol. XIII, Roma, 2002, 491.

J. von HeIN, Die Rezeption US-amerikanischen Gesellschaftsrechts in Deutschland, Tübingen, 2008 (sul quale v., anche, la scheda di F. BORDIGA, in Banca, borsa, tit. cred., 2011, II, 124).

U. HÜFFER, Aktiengesetz ${ }^{10}$, München, 2012, $\$ 1, R d n .22,8$.

P. IVALDI, In tema di applicazione giudiziale del diritto straniero, in Riv. dir. int. priv. e proc., 2010, 585

J. KOHLER, Über die Interpretation von Gesetzen, in GrünbutZ, 1886, 37.

A. La Mattina, Il giudice italiano e il diritto societario straniero, in Dir. comm. int., 2009, 933.

M. LIBERTINI, Diritto civile e diritto commerciale. Il metodo del diritto commerciale in Italia, in Riv. soc., 2013, 1.

P.G. MOnATERI e A. SOMma, L'uso del diritto comparato come interpretazione analogica ex art. 12 preleggi, in Foro it., 1999, V, 48.

A. Pizzorusso, Delle fonti del diritto Art. 1-92, in Commentario del codice civile ScialojaBranca, Bologna-Roma, 2011.

G.B. PORTALE, Società a responsabilità limitata senza capitale sociale e imprenditore individuale con "capitale destinato" (capitale sociale quo vadis?), in Riv. soc., 2010, 1237; ID., Amministrazione e controllo nel sistema dualistico delle società bancarie, in Riv. dir. civ., 2013, I, 25; ID., Le nuove norme sui conferimenti in natura nelle s.p.a.: dal «ravvicinamento» alla «disarmonizzazione» dei diritti nazionali, in Riv. soc., 2012, 11. 
F. RANIERI, Die Rechtsvergleichung und das deutsche Zivilrecht im 20. Jabrbundert: eine Wissenschaftshistorische Skizze, in Das Europäische Privatrecht des 19. und 20. Jabrbunderts, Berlin, 2007, 167; ID., Doctrine et jurisprudence étrangères dans le developpement du droit civil allemande du 20ième siècle, in European Review of Private Law, 2006, 685.

P. ReSCIGNO, Introduzione, in Il giudice e l'uso delle sentenze straniere, cit.

G. SMORTO, L'uso giurisprudenziale della comparazione, in Europa e dir. priv., 2010, 223.

A. Somma, L'uso giurisprudenziale della comparazione nel diritto interno e comunitario, Milano, 2001, 16, 50, 257; ID., Metodi e scopi della comparazione giuridica nelle decisioni delle corti, in Il giudice e l'uso delle sentenze straniere, cit., 97.

Ch. THOLE, Anwendung und Revisibilität ausländischen Gesellschaftsrechts in Verfabren vor deutschen Gerichten, in ZHR, 2012, 15.

F. WIEACKER, Privatrechtsgeschichte der Neuzeit ${ }^{2}$, Göttingen, 1967, 128.

E. Zitelmann, Aufgaben und Bedeutung von Rechtsvergleichung, in Deutsche JuristenZeitung, 1900, 329.

A. ZopPINI (a cura di), La concorrenza tra ordinamenti giuridici, Roma-Bari, 2004.

K. ZwEIGERT, Rechtsvergleichung als universale Interpretationmethode, in RabelsZ, 1949/50, 5; ID., Zur Methode der Rechtsvergleichung, in Studium generale, 1960, 193

\section{Giurisprudenza:}

Corte di Giustizia europea: «Centros», 9 marzo 2009: Causa C-212/97;

«Überseering», 5 novembre 2002: Causa C-208/00; «Inspire Art Ltd»: Causa C-167/01; Cass. - sez. lav., 5 giugno 2009, n. 13087; Cass., 9 maggio 2007, n. 1059; Cass., 26 febbraio 2002, n. 2791; Trib. Padova, 16 maggio 2011; Trib. Venezia, 8 marzo 2011; Trib. Udine, 3 marzo 2009: tutte in Banca, borsa, tit. cred., 2012, II, 222; Bundesgerichtshof, 27 aprile 1976, in NJW, 1976, 1589. 